\title{
La réception de l'œuvre simonienne en Italie
}

\section{Anna Bucarelli}

\section{OpenEdition}

Journals

Édition électronique

URL : http://journals.openedition.org/ccs/828

DOI : $10.4000 /$ ccs. 828

ISSN : 2558-782X

\section{Éditeur :}

Presses universitaires de Rennes, Association des lecteurs de Claude Simon

\section{Édition imprimée}

Date de publication : 31 décembre 2011

Pagination : 177-208

ISBN : 9782354121464

ISSN : 1774-9425

\section{Référence électronique}

Anna Bucarelli, "La réception de l'œuvre simonienne en Italie», Cahiers Claude Simon [En ligne], 7 | 2011, mis en ligne le 21 septembre 2017, consulté le 01 mai 2019. URL : http:// journals.openedition.org/ccs/828; DOI : 10.4000/ccs.828 


\title{
La réception de l'œuvre simonienne en Italie
}

\author{
Anna BUCARELLI \\ Université « Carlo Bo » d'Urbino
}

Hélas, la culture française ne séduit pas : c'est par cette formule, qui exprimait à la fois un regret et la constatation d'une réalité de fait, que Giovanni Bogliolo titrait en 1995 un de ses articles de presse $^{1}$. Le critique y dressait un bilan amer de la réception de la littérature française en Italie, en citant notamment le nom de Claude Simon. «Ce qui est inquiétant, écrivait-il, c'est que, une fois terminé l'éphémère interrègne des deux Marguerite, Duras et Yourcenar, il n'y ait qu'une poignée de lecteurs pour des écrivains comme Tournier et Le Clézio, que le roman posthume de Camus soit accueilli avec une suffisance distraite et que des livres mémorables comme les Lettrines de Gracq et L'Acacia de Claude Simon passent pratiquement inaperçus."

Le jugement, exprimé voici plus de quinze ans, conserve toute son actualité, en particulier pour Claude Simon, un auteur qui n'a jamais touché un large public en Italie, même après l'obtention du prix Nobel, en 1985, et son titre de docteur honoris causa de l'Université de Bologne, décerné en 1989. Comme en témoignent les articles de presse, qui se font l'écho des réserves du public italien, les

1. Giovanni Bogliolo, "Hélas, la cultura francese non seduce ", Tuttolibri, 21 janvier 1995 , p. 1 . Toutes les traductions des textes italiens sont miennes. 
œuvres de l'écrivain sont réputées difficiles. Malgré la qualité des traductions disponibles, malgré l'enthousiasme des interprètes et des critiques qui ont contribué à faire connaître son œuvre, Claude Simon reste, en Italie, un auteur confidentiel.

\section{Claude Simon en italien : les traductions}

Les œuvres simoniennes que les Italiens ont pu lire jusqu'à présent dans leur langue, parfois avec plusieurs années de retard par rapport aux Français, sont au nombre de onze seulement. L'Herbe (L'erba) ouvre la série en 1961, suivie, dans la même décennie, par La Route des Flandres (La strada delle Fiandre) et Le Palace (Il palace). Les années 1970 voient paraître Histoire (Storia) et Triptyque (Trittico), tandis que, dans les années 1980, le prix décerné par l'Académie de Suède suscite la publication de La Chevelure de Bérénice (La chioma di Berenice) et de La Bataille de Pharsale (La battaglia di Farsalo), traduite treize ans après sa parution en France. C'est à la même époque que la maison UTET publie une nouvelle édition de La Route des Flandres et d' Histoire, dans une collection consacrée aux prix Nobel. Enfin, les années 1990 voient paraître les quatre dernières traductions : L'Invitation (L'invito), Discours de Stockholm (Discorso di Stoccolma), L'Acacia (L'acacia) et, après vingt-quatre années d'attente, Leçon de choses (Il senso delle cose) ${ }^{2}$. La chronologie des traductions montre que, jusqu'à l'obtention du prix Nobel, les Italiens ne connaissaient que cinq romans simoniens et ne disposaient pas de certains textes emblématiques: La Bataille de Pharsale, Orion aveugle, Les Corps conducteurs, Leçon de choses et Les Géorgiques. À l'exception de La Bataille de Pharsale et de Leçon de choses, ces œuvres sont, aujourd'hui encore, inaccessibles, ce qui est aussi le cas de romans plus récents comme Le Jardin des plantes et Le Tramway.

2. Chez Einaudi (Turin) ont été publiés : L'erba (1961) ; La strada delle Fiandre (1962 ; 1985) ; Il palace (1965) ; Storia (1971) ; Trittico (1975) ; La battaglia di Farsalo (1987) ; L'acacia (1994) ; Discorso di Stoccolma (1994). Les titres qui complètent la liste sont : La chioma di Berenice, Padoue, Liviana, 1986 ; Le opere. La strada delle Fiandre. Storia, Turin, UTET, 1989 ; L'invito, Brescia, L'Obliquo, 1992 ; Discorso di Stoccolma, Pescara, Tracce, 1992 ; Il senso delle cose, Turin, Testo \& Immagine, 1999. 
De Claude Simon, le lectorat italien ne connaît donc que les titres principaux de la production centrale (celle des années 19601980), mais avec des lacunes importantes. Les premières œuvres des années 1940 et 1950 (depuis Le Tricheur jusqu'au Vent), ainsi que les œuvres publiées après L'Acacia lui sont inconnues. Si l'on pouvait écrire, en 1987, que les lecteurs de Claude Simon avaient « le droit (et la patience) d'attendre " une traduction des Géorgiques ${ }^{3}$, si l'on espérait encore, en 1999, que " quelque éditeur italien se rend[e] compte que Le Jardin des plantes [...] est un des rares livres mémorables de cette fin de siècle $"^{4}$, ces espoirs sont aujourd'hui déçus. Alors que le discours critique affiche un intérêt croissant pour Claude Simon, les traductions se sont arrêtées à la fin des années 1990 : à ce silence éditorial de plus de dix ans, il faut ajouter l'épuisement des traductions existantes, dont seulement deux ou trois sont actuellement disponibles en librairie. Quant à la diffusion de ces textes, nous manquons, pour l'apprécier, de données suffisantes sur les tirages, même si les rééditions attestent le succès de La Route des Flandres et d'Histoire'.

Parmi les éditeurs, Einaudi est certainement celui qui a le plus contribué à faire connaître l'œuvre de Claude Simon au-delà des Alpes. Einaudi fut non seulement le premier éditeur italien, mais aussi le plus fidèle : huit titres furent publiés entre 1961 et 1994. L'anthologie offerte aux visiteurs du Salon du livre de Turin, Il giro del mondo in diciotto autori, témoigne de ce rapport privilégié. Dans ce recueil, les principaux auteurs étrangers de la maison Einaudi sont illustrés par un choix de textes autoréférentiels, composant une galerie d'autoportraits, parmi lesquels figure le Discorso di Stoccolma $a^{6}$.

3. Giovanni Bogliolo, «Simon a Farsalo scompone il tempo in una sinfonia ", Tuttolibri, 21 février 1987, p. 2.

4. Id., "Soldati e muratori in casa di Simon ", Tuttolibri, 24 juin 1999, p. 4.

5. Le premier réédité en 1985 chez Einaudi et en 1989 chez UTET ; le deuxième réédité en 1986 chez Club degli Editori (Milan) et en 1989 chez UTET. On reviendra plus loin sur la fortune des œuvres dans le discours critique.

6. Claude Simon, Discorso di Stoccolma, dans Il giro del mondo in diciotto autori. La narrativa straniera Einaudi 1994 nelle pagine autobiografiche degli scrittori, Turin, Einaudi, 1994. La traduction de L'Acacia, présentée comme le « couronnement d'une longue fidélité ", y est annoncée. 
La contribution des autres éditeurs, plus ponctuelle mais tout aussi significative, fait parfois l'objet d'une déclaration programmatique explicite. C'est le cas de la traduction de Leçon de choses, qui, en 1999, inaugura la collection "Controsegni " des éditions Testo \& Immagine. Le texte, considéré comme exemplaire, servit à lancer un projet éditorial consacré aux « inclassables » du XX ${ }^{\mathrm{e}}$ siècle, ces auteurs qui, bousculant les traditions littéraires et artistiques, expérimentèrent des formes nouvelles à travers les manipulations linguistiques, la subversion des structures narratives et la contamination des arts ${ }^{7}$.

Qu'en est-il, plus généralement, des éléments péritextuels qui orientent et déterminent la réception ? Les traductions se caractérisent, d'une part, par le choix de titres qui reproduisent à la lettre les titres français et, d'autre part, par l'émergence d'un discours éditorial de plus en plus riche. Concernant les titres, la seule exception à la règle de la littéralité est représentée par Leçon de choses, un titre qui « éclaire et justifie " le roman plus que le titre italien Il senso delle cose ("Le Sens des choses "), " car à la traduction littérale, qui conjugue l'idée de lecture avec celle d'instruction, on en a préféré une [...] qui penche de façon inopportune du côté des signifiés " ${ }^{8}$. Par contre, La battaglia di Farsalo, traduction littérale, ne peut pas rendre en italien la lecture anagrammatique du titre français ("La bataille de la phrase »), suggérée par Ricardou et rappelée sur la quatrième de couverture. Quant au discours éditorial, on passe des simples notices bio-bibliographiques des années 1960 à des présentations plus circonstanciées, comme en témoignent les jaquettes de Storia (1971) et de La battaglia di Farsalo (1987). Les textes de ces jaquettes reprennent des extraits des postfaces qui, dans ces deux volumes, analysent certains aspects thématiques et techniques de l'écriture simonienne - nous y reviendrons.

Dans l'ensemble, le péritexte éditorial informe le lecteur sur la vie de l'auteur (son expérience de la guerre ; sa passion pour la peinture et la photographie), sur les romans parus en France, sur leurs traductions italiennes (existantes et à paraître) et sur la réception des œuvres à l'étranger (on évoque le succès obtenu aux États-Unis, en Allemagne et en Angleterre; on cite la presse française ; on rappelle,

7. Cf. "Non è che un inizio ", dans Claude Simon, Il senso delle cose, cit.

8. Giovanni Bogliolo, "Soldati e muratori in casa di Simon ", cit. 
à partir de la deuxième moitié des années 1980, l'attribution du prix Nobel). Le péritexte renseigne aussi sur le contenu thématique des œuvres, soulignant la prédilection simonienne pour les thèmes de l'histoire, du temps et de la mémoire.

Lorsqu'il s'agit de situer l'auteur dans le contexte littéraire français, l'évolution du discours éditorial va de pair avec celle du discours critique, qui problématise de plus en plus le rapport de Claude Simon avec le Nouveau Roman. Après L'erba, présentée en quatrième de couverture comme "la première œuvre traduite en Italie d'une des personnalités les plus riches et les plus complexes du Nouveau Roman français ", les jugements se font plus nuancés : on présente Claude Simon comme l'un des auteurs qui ont été « regroupés de façon plus ou moins arbitraire sous l'étiquette du Nouveau Roman ${ }^{9}$; on le rattache à " l'aile conservatrice " du mouvement ${ }^{10}$; on définit Histoire comme une "expérience fondamentale de synthèse et de dépassement dans le cadre d'une vision romanesque de plus en plus autonome, approfondie et cohérente ${ }^{11}$.

Dès les années 1960, le péritexte identifie des éléments de discontinuité qui permettent de distinguer Claude Simon des autres représentants du Nouveau Roman : une " chaude participation humaine " et " le flux ininterrompu d'un style minutieux, analytique, qui implique les objets, les gestes et les sensations mêmes des personnages " ${ }^{12}$; ou encore la " richesse plus évidente de solutions rhétoriques dans une trame serrée et savamment évocatrice, "faulknérienne" "13. Mais c'est au cours des années suivantes que le discours éditorial mettra surtout l'accent sur l'originalité de son écriture dans le paysage de la littérature contemporaine : Claude Simon n'est plus tellement le continuateur des grandes expériences littéraires du XX ${ }^{\mathrm{e}}$ siècle (Proust, Joyce ou Faulkner ${ }^{14}$ ), mais « un classique, un

9. "Claude Simon Il palace ", note non signée qui se trouve à la fin de Il palace, cit., s.p.

10. Marco Tornar, quatrième de couverture du Discorso di Stoccolma, cit.

11. Quatrième de couverture de Storia, cit.

12. Quatrième de couverture de L'erba, cit.

13. "Claude Simon Il palace », cit.

14. Cités l'un et/ou les autres dans le péritexte éditorial de L'erba, Storia et dans celui de la réédition de La strada delle Fiandre. 
poète de l'écriture, dont l'œuvre a tout à fait droit de cité dans ces régions de l'Éternel que sont la Littérature, la Poésie ${ }^{15}$.

L'étude des péritextes permet de dresser un répertoire des principales caractéristiques de l'écriture simonienne, qui se retrouveront dans le discours critique : "dédoublement du personnage central ; recours au participe présent en tant que signe de simultanéité ou d'action "re-présentée" ; démontage du tissu chronologique ; dialectique entre digression et interruption ; entre logorrhée et réticence ; entre descriptions rapprochées, narrations au ralenti et fuites temporelles " (Il palace), "savante alchimie de temps et de personnages " (Storia), "dynamisme de la description " et " refus des hiérarchies chronologiques" (L'acacia), "style de montage incomparable, technique sophistiquée de composition de lignes thématiques et temps narratifs différents", où ce qui compte "ce ne sont pas les faits ou les personnages, mais un magma en continuelle transformation, le jeu toujours changeant de choses vécues, ou seulement vues, et rangées en souvenir" (La battaglia di Farsalo).

Ces premières analyses, qui anticipent parfois les études spécialisées, s'ajoutent à celles qui portent sur le contenu thématique des œuvres et surtout aux textes critiques qui les accompagnent (nous y reviendrons plus loin). Ainsi, les traductions italiennes, insatisfaisantes du point de vue quantitatif, se présentent-elles avec un péritexte de plus en plus étoffé, qui propose au lecteur des points de repère et des clés d'interprétation.

\section{Voix italiennes de Claude Simon : les traducteurs}

La qualité des traductions, qui sont l'œuvre de dix traducteurs expérimentés, est un autre atout des éditions italiennes. Parmi les traducteurs figurent des écrivains et de grands connaisseurs de l'œuvre simonienne, qui ont doublement contribué à sa diffusion : d'une part, en surmontant les difficultés d'une écriture foisonnante et en la restituant dans une autre langue ; d'autre part, en analysant ses traits spécifiques dans des publications spécialisées, dans la presse et dans le péritexte.

15. Marco Tornar, quatrième de couverture du Discorso di Stoccolma, cit. 
Professeur de littérature française à l'Université de Bologne, Guido Neri, disparu en 1992, fut l'un des premiers traducteurs de Claude Simon. Conseiller éditorial de la maison Einaudi, il a joué un rôle essentiel dans l'introduction en Italie de nombreux auteurs français (outre Claude Simon, il faut citer Antonin Artaud, André Breton, Samuel Beckett et Georges Bataille ${ }^{16}$. On lui doit des traductions remarquables de La Route des Flandres, d'Histoire et de La Chevelure de Bérénice, ainsi que plusieurs textes critiques sur l'œuvre de l'écrivain. La musicalité de la phrase simonienne ${ }^{17}$ est la caractéristique qui inspire son travail de traducteur et qui l'emporte, en particulier, sur la fidélité absolue au participe présent, souvent rendu par l'infinitif, une forme jugée plus maniable en italien. Avec son collègue Liano Petroni, autre éminent professeur de littérature française, disparu en 2006, Guido Neri proposa le nom de Claude Simon pour le titre de docteur honoris causa de l'Université de Bologne, décerné en 1989. L'initiative fut motivée par le parcours littéraire de l'écrivain, sa pratique du roman " comme construction formelle et comme recherche ", le " 'continu' presque musical de blocs verbaux compacts » et le " montage pluritemporel " qui caractérisent son écriture, soulignant sa valeur " non tant comme point extrême d'une crise, mais plutôt comme point de départ, ouverture de nouvelles stratégies de la parole narrative, répondant davantage aux perspectives problématiques élaborées dans notre siècle autour de la mémoire, le temps, la conscience, l'espace, la matière, et le phénomène de la société et de l'histoire $»^{18}$. De cet

16. Un certain nombre de ses préfaces et de ses postfaces ont été réunies dans Esperienze francesi : da Vigny a Leiris, sous la direction de Giancarlo Roscioni et Giulio Ungarelli, Bologne, Pendragon, 1997.

17. " [...] pour moi, la musique de la phrase - j'allais dire de la strophe - de Claude Simon est ce qui compte en tant que traducteur "; " Claude Simon et ses traducteurs européens ", dans Actes des deuxièmes assises de la traduction littéraire (Arles, 1985), Arles, Actes Sud, 1986, p. 102.

18. Voir le "Verbale dell'adunanza del Consiglio della Facoltà di Lettere e Filosofia, 10 settembre 1987 ", dans Atti della Natio Francorum (Bologna, 5-7 ottobre 1989), recueillis par Liano Petroni et Francesca Malvani, Bologne, Clueb, 1993, 2 tomes, I, p. LIII-LV. Texte déjà paru sous le titre " Motivazione del conferimento della Laurea honoris causa a Claude Simon ", Francofonia, X, 18, printemps 1990, p. 15-18. Dans le volume cité on trouve aussi «Claude Simon e la ricerca di una nuova scrittura per il romanzo » (p. LI-LII) par Liano Petroni. 
hommage italien, il nous reste un précieux témoignage : le discours, reproduit ici, où Claude Simon s'interroge sur les " problèmes que posent le roman et l'écriture » en rappelant la phrase de Gustave Lanson qui attribue à l'Italie le mérite d'avoir introduit le goût de l'art dans l'esprit rationnel des Français ${ }^{19}$.

Dans ses articles de revue, dans ses postfaces aux traductions italiennes (Histoire, La Chevelure de Bérénice, La Bataille de Pharsale, $L^{\prime} A$ cacia $^{20}$ ) et dans ses comptes rendus d'essais critiques ${ }^{21}$, Guido Neri analyse les grands thèmes et la syntaxe narrative de l'œuvre simonienne. S’il est impossible de résumer ici le contenu de ces publications, il faut rappeler l'attention constante que Guido Neri réserve à l'intertextualité des œuvres (convergences et divergences qui dessinent le parcours littéraire de l'écrivain), au montage des blocs narratifs (alterné, par 'intersection', collages, marqueteries, structures à contrepoint), aux fonctions ou aux nuances du participe présent dans les différents romans : signe d'une "vanitas vanitatum " de l'action, ou d'" une énergie indifférenciée circulant entre êtres et choses " dans Histoire 22 ; "signe privilégié de la douleur " dans La Chevelure de Bérénice, où " il fixe ce présent, cette actualité, cette simultanéité absolue que nul vécu ne peut atteindre, exaltant le caractère transitoire (à la mort) de toute action $»^{23}$; élément qui participe à l'oscillation entre deuil et mélancolie dans L'Acacia - où, plus qu'ailleurs, il met en œuvre une présence participative du passé

19. Claude Simon, "Problèmes que posent le roman et l'écriture ", dans Atti della Natio Francorum, cit., t. 1, p. LVII-LXII.

20. Guido Neri, "Letture di Histoire », dans Claude Simon, Storia, cit., p. 341362 (traduit en français par Jean Robaey sous le titre " Lectures d' Histoire ", dans Claude Simon, chemins de la mémoire, textes, entretiens, manuscrits réunis par Mireille Calle, Sainte-Foy, Griffon d'Argile, Presses universitaires de Grenoble, 1993, p. 93-102) ; Note à Claude Simon, La chioma di Berenice, cit., p. 60-71 ; "Il romanzo come intersezione ", dans Claude Simon, La battaglia di Farsalo, cit., p. 227-242 ; "Appunti per una lettura dell' Acacia di Claude Simon ", Francofonia, X, 18, printemps 1990, p. 19-27. Rappelons aussi la quatrième de couverture de Trittico, cit., signée par Guido Neri.

21. Ainsi le compte rendu du volume collectif Sur Claude Simon (Éditions de Minuit, 1987) paru dans Francofonia, IX, 16, printemps 1989, p. 125-129.

22. Guido Neri, "Letture di Histoire ", cit., p. 361.

23. Id., Note à Claude Simon, La chioma di Berenice, cit., p.61. 
au présent - condensant " en une ambivalence inextricable [...] ce double effet : fixation mortelle d'un geste en mouvement ou activation d'un dynamisme vital, entre passé et présent $"^{24}$.

Enfin, le nom de Guido Neri a été associé à celui de Claude Simon à l'occasion d'événements culturels d'intérêt international : en 1965, il présente Le Palace avant la remise du prix Formentor ${ }^{25}$; en 1985, il accorde une interview à Paolo Mauri et salue le choix de l'Académie de Suède, en formulant le vœu que Claude Simon ne passe plus pour un auteur difficile ${ }^{26}$.

Professeur émérite et ancien recteur de l'Université d'Urbino, Giovanni Bogliolo est aussi l'auteur de nombreuses traductions de romans et d'essais français (Flaubert, Zola, Cohen, Queneau, Poulet, Tadié, parmi beaucoup d'autres). Par son travail de critique littéraire, il joua également un rôle de médiateur et de divulgateur de l'écriture simonienne. En 1986, Giovanni Bogliolo analysa les procédés qui, dans La Route des Flandres, contribuent à la dimension narrative du "temps supprimé » ou "implosé dans le trou noir de la mémoire ", et qui forgent "une nouvelle façon, conséquente et fascinante, de concevoir le roman $»^{27}$. En 1994, sa traduction de L'Acacia fut saluée par la presse comme une voie idéale pour aborder la production du romancier ${ }^{28}$. À ce travail d'interprète et de traducteur (entreprise ardue, selon ses propres mots $^{29}$ ) s'ajoutent les articles publiés dans la presse, notamment dans "Tuttolibri », le supplément culturel du quotidien La Stampa. Ces articles, souvent associés à un événement éditorial ou à un prix littéraire, ont sans doute contribué

24. Id., "Appunti per una lettura dell' Acacia di Claude Simon », cit., p. 24.

25. Voir Giorgio Zampa, "Si prevedono due vittorie americane al premio Formentor di letteratura ", La Stampa, 1 mai 1965, p. 3.

26. Paolo Mauri, " Provate a leggerlo vi piacerà... ", La Repubblica, 18 octobre 1985 , p. 22.

27. Giovanni Bogliolo, "Il tempo soppresso ne La route des Flandres di Claude Simon ", Bérénice, VII, 16 mars 1986, p. 307-315 ; p. 313 et p. 314. Voir aussi sa préface à Claude Simon, La strada delle Fiandre, Milan, Club degli Editori, 1986, p. V-XIV.

28. Voir, entre autres, Renato Barilli, «Robbe-Grillet e Claude Simon e il racconto fece splash ", Il Corriere della Sera, 6 septembre 1994, p. 27, et Paola Decina Lombardi, "L'acacia folgora il Nobel Simon ", Tuttolibri, 2 juillet 1994, p. 4.

29. Giovanni Bogliolo, "Soldati e muratori in casa di Simon ", cit. 
à faire connaître l'œuvre de Claude Simon à un public plus large, entre les années 1970 et 1990. L'attribution du prix Nobel fut ainsi l'occasion de parcourir la production littéraire de l'écrivain, en décrivant les thèmes et les procédés formels qui font son originalité par rapport aux autres "nouveaux romanciers ", en particulier l'obsession du temps et la présence de l'histoire ${ }^{30}$. Ailleurs, Giovanni Bogliolo nous offre la lecture d'une ouvre parue en traduction italienne, comme La Bataille de Pharsale, pour en dessiner la structure musicale tripartite, avec ses principaux noyaux thématiques, en y reconnaissant " l'esquisse de l'extraordinaire symphonie narrative des Géorgiques " ${ }^{31}$; ou celle de Leçon de choses, considéré comme un texte exemplaire du point de vue de la cohérence du projet narratif simonien, où tout procède de la description : "C'est la force d'expansion des images qui crée l'intrigue et ce sont leurs tropismes qui déterminent la structure. $»^{32}$ Les articles de presse de Giovanni Bogliolo font aussi le point sur la réception italienne de l'œuvre de Claude Simon - nous y reviendrons plus loin.

Parmi les traducteurs italiens de Claude Simon figurent aussi des écrivains. Ainsi Bruno Fonzi (1914-1976), conseiller éditorial chez Einaudi et Garzanti, romancier et nouvelliste, qui consacra sa vie à l'écriture et à la traduction (avec un corpus extrêmement varié d'auteurs anglais, classiques et contemporains, et quelques auteurs français, dont Jean-Paul Sartre et Simone de Beauvoir). C'est à lui que revient le mérite de la première traduction italienne de Claude Simon : L'erba, publiée en 1961. La version italienne de Triptyque porte également une signature illustre : celle de l'écrivain Nanni Balestrini, un des représentants de la néo-avant-garde italienne des années 1960, auteur de poésies expérimentales et de romans engagés sur les mouvements de contestation et les années de plomb (quelques-uns sont traduits en français). Nanni Balestrini partage avec Claude Simon la passion des arts visuels, ainsi qu'une conception du faire littéraire comme incessante expérimentation de formes nouvelles. Ce n'est donc pas un hasard si son nom est associé à celui de Claude Simon

30. Id., "Simon, il romanzo come avventura », La Stampa, 18 octobre 1985, p. 5. 31. Id., "Simon a Farsalo scompone il tempo in una sinfonia ", cit.

32. Id., "Soldati e muratori in casa di Simon ", cit. 
parmi les « inclassables » du XX $\mathrm{X}^{\mathrm{e}}$ siècle, dans le programme éditorial de la collection "Controsegni ", cité plus haut, ou si, dans la postface de Il senso delle cose, ouvrage publié dans la même collection, son recueil Blackout (1980), «l'expérimentation la plus réussie de 'sérialité littéraire' jamais tentée ", est considéré comme "l'exemple littéraire le plus proche de Leçon de choses (malgré la diversité formelle et de contenu) ${ }^{33}$. C'est à Sergio Atzeni, disparu en 1995, que l'on doit la traduction du Discours de Stockholm parue dans l'anthologie Il giro del mondo in diciotto autori. Auteur de romans, de nouvelles et de poésies, Sergio Atzeni a traduit en italien des œuvres de Stendhal, de Patrick Chamoiseau et de Gérard Genette.

Enfin, il faut aussi mentionner le précieux travail de Gioia Zannino Angiolillo, traductrice du Palace (mais aussi de Flaubert, Maupassant, Ionesco, Green, Ollier, Sarraute, Semprun et Duras) ; de Dianella Selvatico Estense, qui a traduit La Bataille de Pharsale (mais aussi des œuvres de Maupassant, Radiguet, Le Clézio, Sagan, Beauvoir, Perec, Duras et Colette) et qui est aussi l'auteur de plusieurs recueils de poésies ; de Marco Ferri, traducteur de Victor Hugo et poète lui-même, à qui l'on doit la traduction de L'Invitation et la Nota qui l'accompagne ${ }^{34}$ (où il relève la manière iconique de formuler la réflexion politique ou historique) ; d'Anna Maria Salvatore, qui a signé la traduction du Discours de Stockholm parue en 1992 (et d'une pièce de Jean Giraudoux) ; enfin, de Roberto Marro, créateur de la collection "Controsegni " (où il a traduit aussi des ouvres de Robbe-Grillet), qui a signé la version italienne de Leçon de choses; sa postface critique retrace la genèse du texte et analyse sa structure musicale en termes de sérialité, en le rapprochant des expériences poétiques de Nanni Balestrini.

\section{Claude Simon dans la presse italienne}

Les articles de presse consacrés à Claude Simon sont associés à deux moments importants : la consécration du prix Nobel, en oc-

33. Roberto Marro, "La musica delle cose », dans Claude Simon, Il senso delle cose, cit., p. 139, note 3 .

34. Marco Ferri, « Nota », dans Claude Simon, L’invito, cit., p. 45. 
tobre 1985, et l'hommage rendu à l'écrivain après sa mort, en juillet 2005. Les autres articles suivent la parution des œuvres en France et en Italie, témoignant de sa fortune dans les deux pays, et proposant parfois des interviews.

En 1985, après s'être fait l'écho des rumeurs précédant l'attribution du prix $\mathrm{Nobel}^{35}$, la presse rendit compte des réactions de surprise, nuancées de réserves, qui accueillirent le lauréat, pourtant considéré comme «nobélisable » depuis longtemps. Pour Guido Neri, l'effet de surprise s'explique par le fait que "Simon, du point de vue biographique, est une figure presque inexistante ", ayant toujours essayé de gommer " toute référence biographique, toute contamination entre l'homme et l'œuvre ${ }^{36}$. De son côté, Carlo $\mathrm{Bo}^{37}-$ grand intellectuel, grand connaisseur de la littérature française et recteur de l'Université d'Urbino pendant plus de cinquante ans - met l'accent sur les polémiques qui accompagnent toujours la décision de l'Académie de Suède, non sans prévoir une double protestation (de la part des pays exclus et de la part du pays favori) et l'objection rituelle : il y avait quelqu'un d'autre à prendre en considération (en l'occurrence, René Char). Il rend compte aussi des principales réserves - en particulier, le caractère élitiste et exigeant de l'écriture simonienne exprimées dans la plupart des articles de presse, qui font de Claude Simon un auteur réputé difficile et inconnu du grand public ${ }^{38}$. Pour contester le choix de l'Académie de Suède, d'autres regrettent qu'une femme (Marguerite Yourcenar ou Marguerite Duras) ${ }^{39}$ n'ait pas été consacrée ; d'autres encore estiment que, pour Claude Simon, la consécration vient trop $\operatorname{tard}^{40}$ - dans une interview publiée en 1989, l'écrivain rappelle pourtant le caractère expérimental de ses romans,

35. "A Claude Simon il Nobel letterario ? ", La Stampa, 17 octobre 1985, p. 5.

36. Paolo Mauri, " Provate a leggerlo vi piacerà... ", cit.

37. Carlo Bo, "A Claude Simon il premio Nobel ed è polemica ", Il Corriere della Sera, 18 octobre 1985 , p. 7.

38. Cf. Elena Guicciardi, "Il Nobel a Simon », La Repubblica, 18 octobre 1985, p. 22 et "Simon : io tento di descrivere quel magma che è la vita mentale ", Tuttolibri, 19 octobre 1985 , p. 1.

39. Milena Milani, "Donne scrittrici grandi e ignorate ", Stampasera, 21 octobre 1985 , p. 3.

40. Jean-Paul Aron, "Simon, un Nobel fuori tempo ", Il Corriere della Sera, 10 décembre 1985 , p. 1. 
adressés " aux générations futures » ${ }^{41}$. La thèse du prétendu retard fut dénoncée à la fois par Guido Neri - pour qui l'expérimentalisme de Simon " ne se rattache pas à la dernière époque, mais [...] à la grande époque du XXe siècle des Joyce, des Proust et des Faulkner " ${ }^{42}-$ et par le poète, romancier et critique littéraire Edoardo Sanguineti, qui voit dans " cette reconnaissance, tardive mais bien méritée, d'une écriture problématique [...] une occasion qu'il serait inique de gaspiller » et qui se propose de surmonter les polémiques retraçant l'itinéraire du lauréat, car "si l'on considère le travail et la vie de Simon, le choix de l'Académie suédoise [...] apparaît moins discutable ${ }^{43}$.

Au-delà des réserves, les articles publiés à cette occasion offrent au lecteur une vue d'ensemble sur la production simonienne à travers des notices bio-bibliographiques, des anciennes interviews parues en France ${ }^{44}$ et des extraits de romans, mais aussi des mises au point sur le 'nouveau romancier' et des aperçus sur sa poétique et sa pratique littéraire. Ainsi Elena Guicciardi ${ }^{45}$ reprend la division de l'œuvre en trois mouvements, proposée par John Fletcher, tandis que Giovanni Bogliolo parcourt l'itinéraire de l'écrivain à partir de l'« équivoque » qui a permis de l'assimiler au Nouveau Roman après la parution du Vent et de L'Herbe à cause de la "description minutieuse, l'accumulation de faits incongrus et souvent dérisoires » du premier et de cet "effet optique ", encore plus visible dans le second, qui fait que "tous les événements concrets reculent vers le fond, tandis que le premier plan est envahi par leur alternance, leur succession et leur enchevêtrement ${ }^{46}$. Revenant sur la même 'équivoque', Edoardo Sanguineti observe que la présence obligée de Claude Simon au sein du Nouveau Roman procède d'une "nécessité d'exhaustivité documentaire plus que d'un plein consente-

41. "Parla Simon. E così rischiai di perdere all'ultimo istante ", Intervista di Gaia Giorgetti, Il Messaggero, 18 octobre 1989.

42. Paolo Mauri, "Provate a leggerlo vi piacerà... ", cit.

43. Edoardo Sanguineti, "Un occhio da Nobel », L'Unità, 18 octobre 1985, p. 13.

44. Dans "Simon : io tento di descrivere quel magma che è la vita mentale ", cit., on trouve deux interviews, l'une parue dans Le Monde en 1967 à la sortie d'Histoire et l'autre parue quelques années auparavant dans La Quinzaine littéraire.

45. "Il Nobel a Simon", cit.

46. Giovanni Bogliolo, «Simon, il romanzo come avventura », cit. 
ment ", tout en soulignant que si l'on peut parler d'une 'école du regard' " personne n'a joué avec autant de lucidité la part de l'œil dans la construction romanesque $»^{47}$. Son article au titre éloquent, "Un occhio da Nobel " (" Eil de Nobel ») insiste sur l'importance du regard comme " principe structurant " qui détermine "le glissement épiphanique des sections de réalité " dans l'espace et le temps et en tant que mode perceptif « d'une réalité cassée, d'un cosmos fait chaos " selon un "réalisme qui ne repose pas sur l'analyse maniaque et hallucinatoire d'objets et figures, mais sur ce sentiment de désastre qui se réfléchit dans tout fragment de son écriture ainsi que dans le corpus global " de son œuvre. De son côté, Carlo Bo dégage le côté intellectuel et philosophique de l'œuvre, non sans évoquer l'"Odyssée sans Ulysse " ou la "navigation vers le rien " qui définit le récit simonien de la mémoire, "comme si la fameuse 'Madeleine' proustienne, ici de proportions gigantesques, était explosée et que les fragments de cette infinie cassure restaient comme les signes et les symboles d'une impossible reconstruction ».

En juillet 2005, les journaux italiens rendent hommage à l'écrivain disparu, proposant d'autres raccourcis et commentaires sur sa longue expérience littéraire. Il suffit de lire les titres pour y retrouver, condensés en formules, les enjeux majeurs de sa poétique : la centralité du regard dans "Simon, lo sguardo che riscrisse il mondo ${ }^{48}$; l'expérimentation constante de son écriture dans « L'anti romanzo di Simon ${ }^{49}$; le caractère à la fois baroque et réaliste de son œuvre dans "Barocco, ossessivo, dentro la realtà " ${ }^{50}$; la perception sensorielle de la réalité dans "Claude Simon. L'antiromanziere sensoriale " ${ }^{51}$; le refus d'une poétique de l'inspiration dans "Ispirazione ? No grazie " ${ }^{52}$; la défense de l'artisanat de l'écriture et de son autonomie dans «Si-

47. Edoardo Sanguineti, art. cit.

48. Paola Decina Lombardi (La Stampa, 10 juillet 2005, p. 28-29).

49. Daria Galateria (La Repubblica, 11 juillet 2005, p. 43).

50. La page ainsi intitulée comprend l'article de Giuliana Morandini, "Quel girino chiamato io ", et un portait de l'écrivain-viticulteur, "Ma sono un viticultore ", signé par Piero Vigorelli (Il Messaggero, 18 octobre 1985, p. 7).

51. Idolina Landolfi (Il Giornale, 10 juillet 2005, p. 25).

52. Giuseppe Scaraffia (Il Sole 24 Ore, suppl. du dimanche, 10 juillet 2005, p. 33). 
mon, paladino della scrittura $»^{53}$; la représentation du chaos dans "Addio a Claude Simon, il romanziere del disordine del mondo ${ }^{54}$.

Certains articles abordent aussi la composition des romans, comme le montage plurichromatique de La Route des Flandres, en rappelant la constante recherche d'un ensemble harmonique à l'image des compositions picturales. On cite également des extraits de son Discours de Stockholm et de La Route des Flandres, ainsi que la motivation du prix Nobel, et on se souvient des prix obtenus par ses œuvres et de l'engagement de l'homme en tant que signataire du Manifeste des 121 et d'autres appels comme celui contre la course aux armements en 1983 ou, en 1996, contre l'exploitation sexuelle des enfants.

En ce qui concerne les articles qui s'échelonnent sur plusieurs décennies, on peut relever que le nom de Claude Simon fait ses premières apparitions dans la presse des années 1960 parmi les représentants du Nouveau Roman ${ }^{55}$, n'occupant souvent que le second rang, derrière Michel Butor, Nathalie Sarraute et surtout Alain Robbe-Grillet - dont Renato Barilli a introduit en Italie les essais théoriques au début de la décennie. L'écrivain attire aussi l'attention de la presse à l'occasion de la remise de prix littéraires, français ou internationaux : le prix Médicis de $1967^{56}$; le prix Formentor de 1963 et $1965^{57}$; enfin le prix Nobel, Claude Simon étant déjà cité parmi les lauréats possibles en 1968 et $1969^{58}$. Aux notices des prix s'ajoutent, dans les années 1960 et 1970, des articles qui rendent compte des romans publiés en France ou de leurs traductions italiennes, tout en dégageant l'originalité de l'auteur. Ainsi, lors de la

53. Giovanni Mariotti (Il Corriere della Sera, 10 juillet 2005, p. 31).

54. M.S.P. (L'Unità, 10 juillet 2005, p. 23).

55. Citons, par exemple, Franco Simone, "Tradizione ed originalità del nuovo romanzo francese ", La Stampa, 19 août 1964, p. 11.

56. "Il romanzo di un'operaia riceve il premio Fémina ", La Stampa, 28 novembre 1967, p. 3.

57. Cf. "Lo scrittore Gadda e Ludovico Terzi proposti per il Formentor ", Stampasera, 29 avril 1963, p. 5 et Giorgio Zampa, art. cit.

58. Voir "Oggi sarà assegnato il premio Nobel 1968 ", La Stampa, 17 octobre 1968, p. 15, "Il poeta Ungaretti vincerà il Nobel ? ", Stampasera, 20 octobre 1969, p. 5 et Sandro Volta, "Il Nobel ad André Malraux ? ", La Stampa, 11 octobre 1969 , p. 15. 
publication en France d'Histoire, Claude Simon est présenté comme l'écrivain du Nouveau Roman "qui a créé l'œuvre la plus solide et la plus parfaite ", une œuvre qui exige du lecteur une collaboration " non seulement intellectuelle, mais aussi sensorielle "59 ; et quand paraissent les traductions italiennes d'Histoire et de Projet pour une révolution à New York, un critique souligne la " confluence occasionnelle " des nouveaux romanciers pour inviter le lecteur à "suivre les développements toujours plus divergents d'un parcours destiné à subir encore d'infinies diversions ${ }^{60}$. Mais c'est surtout après l'obtention du prix Nobel, en 1985, que se multiplient les articles, parfois accompagnés d'interviews ${ }^{61}$. La presse italienne s'occupe des derniers romans, L'Acacia, Le Jardin des plantes et Le Tramway ${ }^{62}$, ou

59. Ugo Ronfani, "Claude Simon rifiuta di trattare i personaggi come i suoi vigneti ", La Gazzetta del Popolo, 17 mai 1967, p. 3.

60. Giovanni Bogliolo, "Nouveau Roman oggi ", La Stampa, 18 février 1972, p. 14.

61. Parmi les interviews parues dans la presse italienne, il faut citer : "Il contadino e il soldato. Intervista con Claude Simon di Karin Holter" (Lettera internazionale, n. 7, 1986, p. 54-55), qui aborde, à partir des Géorgiques, la conception simonienne de l'histoire ; celles réalisées, en 1989, par Paola Decina Lombardi ("Simon a colori ", Tuttolibri, 28 octobre 1989, p. 6) pour le titre de docteur honoris causa décerné à l'écrivain, qui s'exprime sur L'Acacia et sur l'influence du cubisme dans sa vision de la réalité et par Gaia Giorgetti, "Parla Simon. E così rischiai di perdere all'ultimo istante », cit. ; enfin, l'interview d'Enrico Regazzoni ("Claude Simon Nobel difficile ", La Repubblica, 22 juillet 1992, p. 32), où Simon dresse le portrait de l'écrivain en 'Orion aveugle' et formule sa définition de la crédibilité du roman en termes (musicaux) d'assonances et dissonances. De Claude Simon a paru aussi en Italie "Roman, description et action ", Studi di letteratura francese, VIII, 1982, p. 12-27 (conférence prononcée à l'occasion du 45 Symposium des Prix Nobel tenu 1978) ; un autre petit texte, qui reprend le passage du Discours de Stockholm consacré à Balzac, est "À propos de Balzac / A proposito di Balzac ", dans Paola Decina Lombardi, Mosaico Balzacchiano. La donna e la norma nella Commedia umana, con una nota di Claude Simon e un'appendice di testi e documenti, Naples, Edizioni scientifiche italiane, 1991, p. 6-7.

62. Le premier présenté par Elena Guicciardi, "Sotto l'acacia Claude », La Repubblica, 26 septembre 1989, p. 33 ; le deuxième par Fabio Gambaro, "Torna in libreria il Nobel Claude Simon ", La Repubblica, 20 septembre 1997, p. 38 et par Gabriella Bosco, "Nel giardino di Simon la vita è un teorema ", Tuttolibri, 2 octobre 1997, p. 3 ; le troisième par Fabio Gambaro, «In tram con il Nobel Claude Simon ", La Repubblica, 27 avril 2001, p. 44. 
des traductions italiennes de La Bataille de Pharsale, de L'Acacia et de Leçon de choses $^{63}$. La présentation des œuvres comprend des observations sur la structure romanesque, sur les principaux noyaux thématiques, sur la matière familiale et historique, sur les références iconographiques et littéraires, avec parfois une exposition de la trame à la façon d'un narrateur traditionnel. Certains articles commentent aussi la réception des œuvres en France et en Italie, en tentant d'estimer la fortune de la production simonienne. En ce qui concerne les parutions françaises, la presse italienne évoque le succès obtenu par L'Acacia, "salué par la critique comme un chef-d'œuvre » et " rangé dès sa sortie parmi les best-sellers ${ }^{64}$, par Le Jardin des plantes, considéré comme "l'un des événements majeurs de l'année littéraire ", qui confirme Claude Simon " comme l'un des plus grands auteurs de la littérature française du XX $\mathrm{X}^{\mathrm{e}}$ siècle, et un maître de l'alchimie de la mémoire "65, et par Le Tramway "dont toute la presse a souligné la qualité, la profondeur et la rigueur du style " ${ }^{66}$. Par contre, les articles qui concernent les traductions italiennes offrent des bilans qui sont loin d'être triomphaux. Ainsi, en 1987, à la sortie de la traduction de La Bataille de Pharsale, déjà annoncée par Einaudi en 1975, Giovanni Bogliolo observe que, sans le prix Nobel, la maison d'édition "n'aurait pas tenu, avec douze ans de retard, sa promesse, et il est certain que bien peu de lecteurs s'en seraient plaints ou seulement rendus compte ». Et le critique de rappeler que si "Simon a pu compter dès le début sur une poignée sélectionnée et aguerrie de spécialistes et exégètes éparpillés dans le monde [...] il n’a jamais réussi, même pas en France, à toucher le grand public $»^{67}$. En effet, malgré le prix Nobel et le titre de docteur honoris causa de l'Uni-

63. Pour la première voir Giovanni Bogliolo, «Simon a Farsalo scompone il tempo in una sinfonia ", cit. ; pour la deuxième Renato Barilli, "Robbe-Grillet e Claude Simon e il racconto fece splash », cit. et Paola Decina Lombardi, "L'acacia folgora il Nobel Simon ", cit. ; pour la troisième Giovanni Bogliolo, "Soldati e muratori in casa di Simon ", cit. et Paolo Mauri, "Poveri Nobel quasi sconosciuti ", La Repubblica, 8 mars 1999, p. 30.

64. Elena Guicciardi, "Sotto l'acacia Claude », cit.

65. Fabio Gambaro, "Torna in libreria il Nobel Claude Simon ", cit.

66. Id., "In tram con il Nobel Claude Simon », cit.

67. Giovanni Bogliolo, "Simon a Farsalo scompone il tempo in una sinfonia ", cit. 
versité de Bologne, qui, en 1989, donna l'occasion de revenir sur le travail du romancier et de publier des interviews ${ }^{68}$, les articles de presse des années 1990 insistent sur l'accueil réservé du lectorat italien : Claude Simon est "étiqueté come un auteur "difficile" " ${ }^{69}$ sur qui « pèse un préjugé d'illisibilité que même le Nobel n’a pas réussi à dissiper $»^{70}$. Les titres se font l'écho de ce préjugé, comme dans " Claude Simon Nobel difficile $»^{71}$, et de l'indifférence du public (qui parfois s'étend à la littérature française ${ }^{72}$, comme dans «Claude Simon un Nobel quasi sconosciuto ${ }^{73}$, où Paolo Mauri, à quelques mois de la parution italienne de L'Acacia, " qui a comme sombré dans un vide total ", se propose d'offrir une " modeste réparation " à son auteur par une contestation enflammée de sa prétendue difficulté : "L'Acacia, dans son genre, est même [...] un livre facile. Mais pourvu que son lecteur ne soit pas 'facile'. C'est-à-dire pas facile à contenter. La raison pour laquelle Claude Simon est aujourd'hui oublié chez nous ne réside pas dans la difficulté de son roman, mais dans la facilité à laquelle on s'habitue trop souvent. Peut-être préférant ou, pis encore, exigeant et exaltant une écriture de reportage (journalistique ?), même chez les romanciers ».

\section{Claude Simon et la critique spécialisée}

Entre les années 1950 et 1970, l'œuvre de Claude Simon ne fait pas l'objet d'un examen approfondi dans la critique spécialisée qui est publiée en Italie ${ }^{74}$. Si plusieurs contributions sont consacrées au Nouveau Roman, aucune étude ne porte spécifiquement sur la production simonienne, à deux exceptions près : la postface à Histoire

68. Voir en particulier celle, déjà mentionnée, de Paola Decina Lombardi.

69. Paola Decina Lombardi, "L'acacia folgora il Nobel Simon ", cit.

70. Giovanni Bogliolo, "Soldati e muratori in casa di Simon ", cit.

71. Titre de l'interview déjà mentionnée d'Enrico Regazzoni.

72. Dans l'article cité de Giovanni Bogliolo, "Hélas, la cultura francese non seduce ".

73. Article de Paolo Mauri (La Repubblica, 17 décembre 1994, p. 38) ainsi que "Poveri Nobel quasi sconosciuti ", cit.

74. Nous prenons en compte non seulement les textes critiques publiés en italien, mais tous ceux qui, même écrits en français, ont paru dans des revues et des volumes italiens. 
de Guido Neri, déjà mentionnée, et l'étude pionnière publiée en 1969 par Lucia Poli ${ }^{75}$, qui analyse les romans comme " une phénoménologie en acte ", en référence à Merleau-Ponty.

Si le nom de Claude Simon est absent, en 1962, du Dizionario universale della Letteratura contemporanea ${ }^{76}$, où figurent pourtant Alain Robbe-Grillet et Nathalie Sarraute, il apparaît en 1980 dans un Dizionario della Letteratura mondiale del 900, qui le présente comme l'un des maîtres du Nouveau Roman et un "réaliste du microcosme ». L'article évoque les "descriptions précises jusqu’au byzantinisme ", la " fragmentation, la continuelle dispersion du tissu narratif ", tout en rappelant les polémiques suscitées par l'œuvre, ainsi que les qualifications - "décourageante et irritante " ${ }^{77}$ - qui lui furent parfois appliquées.

C'est à partir des années 1980, en effet, que la critique italienne consacre à Claude Simon des études de plus en plus nombreuses, témoignant de l'intérêt grandissant qui est porté à son œuvre. Arrêtons-nous d'abord aux monographies : cinq au total, qui ne sont pas antérieures aux années 1980. De ces cinq monographies, publiées entre 1982 et 2005, trois seulement se consacrent exclusivement à l'œuvre de Claude Simon : l'une en présente une vision globale ; les autres approfondissent un aspect particulier. Dans Introduzione al romanzo di Claude Simon ${ }^{78}$, publiée en 1993, Manuela Raccanello décrit l'itinéraire de l'écrivain, tandis que Graziano Benelli propose une lecture du Vent qui souligne le rôle décisif de ce roman 'du doute', contenant « en germe tous les procédés narratifs qui carac-

75. «L'esperienza di Claude Simon », Paragone, XX, 230, avril 1969, p. 108-129. 76. Milan, Mondadori, 1962.

77. Giorgio Mirandola, "Simon Claude", dans Dizionario della Letteratura mondiale del 900, dirigé par Francesco Licinio Galati, Milan, Edizioni Paoline, 1980, p. 2759-2760. Parmi les Dictionnaires littéraires qui mentionnent l'œuvre simonienne, on rappellera le Dizionario Bompiani degli Autori di tutti i tempi e di tutte le letterature, Milan, Bompiani, 1987 (et la nouvelle édition parue en 2006), ainsi que le Dizionario Bompiani delle Opere e dei Personaggi di tutti i tempi e di tutte le letterature, Milan, Bompiani, 2005, où figurent cinq romans simoniens : La Route des Flandres, Histoire, La Bataille de Pharsale, Les Géorgiques et L'Acacia.

78. Manuela Raccanello et Graziano Benelli, Introduzione al romanzo di Claude Simon, Trieste, Edizioni del Tornasole, 1993. 
tériseront la future (radicale) production ${ }^{79}$. L'étude de Manuela Raccanello passe en revue les textes simoniens, depuis Le Tricheur jusqu'à L'Acacia, et s'appuie sur les lectures critiques de Dällenbach, de Ricardou et de Duncan pour souligner l'unité, l'évolution stylistique et la continuité thématique de l'univers narratif - " un univers encombré d'objets, dominé par l'entassement baroque de détritus hétérogènes " qui parlent " un langage de décomposition et de mort "-ainsi que la remise en question du récit à travers une écriture au présent, reposant sur la « nature métaphorique de la langue ${ }^{80}$.

De son côté, le volume plus récent de Simonetta Micale ${ }^{81}$, publié en 2005, étudie le rapport entre photographie et écriture. L'auteur analyse l'acte énonciatif qui inspire les Photographies 1937$1970^{82}$, étudie le thème du mur dans les textes littéraires et photographiques $^{83}$, et propose une lecture du Vent qui montre comment le thème de la photographie envahit le roman à tous les niveaux ${ }^{84}$. Pour Micale, il y a continuité et complémentarité - thématiques et poétiques - entre image photographique et écriture romanesque, qui reflètent une même vision du monde.

79. Graziano Benelli, «Il 'vento dello scandalo' ", in Ibid., p. 49-61 ; p. 51.

80. Manuela Raccanello, "Il labirinto e la vertigine ", in Ibid., p. 6-48 ; p. 9 et p. 13.

81. Il fotogramma e la frase. Incontri di fotografia e scrittura nell'opera di Claude Simon, Naples, Edizioni Scientifiche Italiane, 2005.

82. Selon Micale, les photographies, par leur mise en volume et par les titres qui les accompagnent, sont soustraites à la contingence, à la discontinuité et à la fixité temporelle pour être introduites dans la dimension narrative de la continuité et du temps qui coule, reflétant ainsi la dialectique entre ordre et désordre, immobilité et mouvement, qui constitue le souffle de l'œuvre simonienne.

83. Partie qui développe l'étude "Il muro nell'opera di Claude Simon. Immagini fotografiche e immagini letterarie ", dans Lingua, cultura e testo. Miscellanea di studi francesi in onore di Sergio Cigada, sous la direction d'Enrica Galazzi et Giuseppe Bernardelli, Milan, Vita e pensiero, 2003, t. II/2, p. 847-862. Relevant dans l'univers de Simon l'absence du mur en tant qu'élément de division, Micale analyse surtout sa fonction de dépositaire des traces du passé par accumulation ou par juxtaposition.

84. Notamment à travers un protagoniste-photographe qui, par son regard acritique, atemporel et déprédateur qui méduse la réalité, devient un symbole de l'art photographique. 
Enfin, la monographie de Bérénice Bonhomme ${ }^{85}$, publiée elle aussi en 2005, propose également une approche intersémiotique : l'auteur étudie le rapport de l'écrivain à l'image cinématographique en analysant le court-métrage que Claude Simon réalisa en 1975 en adaptant son roman Triptyque. Cette confrontation entre le romancier et le cinéaste, entre le langage verbal et les images (fixes et mobiles), fait ressortir leur synergie dans la création d'un nouveau langage.

Les deux autres monographies, qui sont consacrées au Nouveau Roman, analysent les œuvres de Claude Simon dans leur contexte, tout en dégageant les traits spécifiques de son écriture. La première, publiée de façon pionnière par Mireille Calle-Gruber en 1982, explore la figure du labyrinthe dans l'univers narratif de Michel Butor, d'Alain Robbe-Grillet et de Claude Simon ${ }^{86}$, trois auteurs traduits en italien, et qui ont élaboré une réflexion critique sur leur projet littéraire. Loin de signifier une impasse, le labyrinthe se révèle un " symbole de la création littéraire " qui " participe d'une détermination politique : celle qui essaie, contre l'idéologie dominante [...] d'enraciner la fiction dans la matérialité de l'écriture et d'ouvrir ainsi la voie à une lecture différente : active et créatrice ${ }^{87}$. La seconde monographie, publiée par Simonetta Micale en $1995^{88}$, s'intéresse aux virtualités narratives dans le Nouveau Roman. Le chapitre consacré à Claude Simon analyse les procédés narratifs de la virtualité simonienne à partir de trois aspects fondamentaux : le roman comme com-position ou réseau de relations possibles, la modalité d'autogénération de l'écriture et la narration hypothétique.

85. Bérénice Bonhomme, Triptyque de Claude Simon. Du livre au film. Une esthétique du passage, Fasano, Schena / Paris, Presses de l'Université de Paris-Sorbonne, 2005.

86. Itinerari di scrittura : nel labirinto del Nouveau Roman. M. Butor, A. RobbeGrillet e C. Simon, Rome, Bulzoni, 1982.

87. Ibid., p. 127. À partir de l'expérience du labyrinthe des personnages, on considère son élévation à symbole de la condition humaine, la transformation du mythe de Thésée en une condition odipienne de douloureuse descente en soimême, d'où l'on regagne la surface grâce au jeu du langage, pour aboutir enfin aux procédés labyrinthiques de l'écriture.

88. Simonetta Micale, Se il conte non fosse stato innamorato. Virtualità narrative nel "nouveau roman », Reggio Emilia, Diabasis, 1995. 
En ce qui concerne les essais publiés en revue ou en volume, il faut distinguer ceux qui portent sur l'ensemble de la production et ceux qui s'occupent d'une œuvre en particulier. La monographie de Pierluigi Ligas, qui appartient à la première catégorie, nuance en quelques pages l'image d'un auteur qui aurait rompu définitivement avec le roman traditionnel ( il ne se révèle ni trop intellectuel pour ne 'voir' que les idées laissant de côté les hommes, ni trop poète pour se détacher de la réalité ${ }^{89}$ ) et suggère de le comparer à l'écrivaine italienne Elsa Morante pour sa conception de l'histoire. Dans l'étude d'ensemble qui accompagne l'édition UTET de Storia et de La strada delle Fiandre ${ }^{90}$, Anna Paola Mossetto passe en revue la production simonienne, des premiers romans à L'Invitation. Son analyse met en lumière la matière généalogique qui les informe, ainsi que les modèles géométriques et plastiques qui agissent aux niveaux sémantique et structurel. Dans une autre étude sur le Nouveau Roman, Anna Paola Mossetto Campra ${ }^{91}$ décrit l'histoire du mouvement, commente ses interprétations critiques et s'appuie sur l'œuvre de Claude Simon pour souligner le rejet de la psychologie (reconnaissable dans les premiers romans) et le choix d'un formalisme de plus en plus radical.

Parmi les essais thématiques qui embrassent l'ensemble des œuvres, il faut citer celui de Graziano Benelli, consacré à la figure du soldat, "emporté vers une dégradation irréversible qui le verra se dissoudre dans les éléments de la Nature ${ }^{92}$, et celui de Robin

89. Pierluigi Ligas, "L'avventura del racconto ", in Id., Microcosmi. Da AlainFournier à Claude Simon. Sguardi critici tra biografie e opere, Vérone, Libreria Universitaria, 1992, p. 116-121; p. 119.

90. Anna Paola Mossetto, "Claude Simon », in Claude Simon, Le opere. La strada delle Fiandre. Storia, cit., p. IX-XXXIII ; 651-660. Du même auteur voir aussi "Claude Simon ", dans Poeti e narratori francesi del Novecento, sous la direction de Sergio Zoppi, Rome, La Nuova Italia Scientifica, 1987, p. 246-248.

91. "Nouveau Roman ", dans Letteratura Francese Contemporanea. Le correnti d'avanguardia, sous la direction de P.A. Jannini et G.-A. Bertozzi, Rome, Lucarini, 1984, p. 349-417.

92. Graziano Benelli, «La figura del soldato nel romanzo di Claude Simon », dans Parcours et rencontres. Mélanges de langue, d'histoire et de littérature française offerts à Enea Balmas, t. II, "XVIII - XX ${ }^{\mathrm{e}}$ siècle », sous la direction de Paolo Carile, Giovanni Dotoli, Anna Maria Raugei, Michel Simonin, Luigia Zilli, Paris, Klincksieck, 1993, p. 1367-1380 ; p. 1377. 
Lefere, qui étudie les parentés littéraires de l'écrivain, en particulier les éléments narratifs, thématiques et formels qui le rapprochent de Marcel Proust : la logique du texte (analogique), la progression digressive d'une phrase complexe, la problématique du temps, du mouvement et de l'immobilité, des rapports entre les mots-concepts et les choses, la promotion du descriptif et la figuralisation ${ }^{93}$.

Les essais qui portent sur une œuvre particulière sont plus nombreux que les précédents. Six sont consacrés à $\mathrm{La}$ Route des Flandres. Les deux études de Giovanni Bogliolo, déjà citées, considèrent, entre autres aspects, la dimension temporelle du roman, tandis que celle de Pierre Gille, consacrée à la relation entre corruption et génération, montre " comment les modes de corruption mis en ouvre dans le roman peuvent à tout moment renvoyer à l'écriture, comment cette activité correspondante participe d'un jeu de dédoublement général qui interdit de dissocier la génération et la corruption $»^{94}$. Dans son analyse des voix narratives, Anne-Marie Miraglia examine « le double défi inhérent à la construction du texte " : "le défi représenté par la tentative des diverses voix pour saisir et exprimer le réel, le distinguer de l'imaginé et du rêve et, d'autre part, le défi que la multiplication des voix et la fragmentation des récits lancent à la critique narrative ${ }^{95}$. Tandis que Claude Morier propose une lecture sémiotique d'un fragment du roman ${ }^{96}$, Lina Zecchi ${ }^{97}$ voit dans le retard de la traduction italienne le sort paradoxal d'un écrivain nobélisé mais incompris, et définit le roman comme un "mécanisme textuel construit avec une volonté structurante de type mathématique, absolument antinaturaliste et combinatoire ». Quatre essais portent sur Histoire : celui de Guido Neri, déjà cité ; les articles de Jean Rousset et de Giuliana

93. Robin Lefere, "Claude Simon et Marcel Proust ", Studi francesi, n. 100, XXXIV, 1, 1990, p. 91-100.

94. Pierre Gille, "Génération et corruption dans La Route des Flandres de Claude Simon ", Studi francesi, n. 98, XXXIII, 2, mai-août 1989, p. 296-302 ; p. 296.

95. Anne-Marie Miraglia, "La Route des Flandres: défi aux vois narratives ", Studi francesi, n. 101, XXXIV, 2, mai-août 1990, p. 265-271; p. 265.

96. Claude Morier, «Per un commento semiotico dei testi letterari », trad. par S. Hotelier, Strumenti critici, 19, octobre 1972, p. 308-329.

97. Lina Zecchi, compte rendu de Claude Simon, La battaglia di Farsalo, L'Indice, n. 9, 1987. 
Costa-Colajanni, qui interrogent la fonction des cartes postales dans le tissu narratif ${ }^{98}$; et un texte d'Andrea Calì, qui montre la con-fusion entre récit et discours dans un fragment du roman ${ }^{99}$. Deux textes analysent La Bataille de Pharsale : la postface, déjà citée, de Guido Neri et la contribution d'Alberto Casadei sur les sources iconographiques du roman ${ }^{100}$. Deux essais sont consacrés à Leçon de choses: la postface de Roberto Marro, déjà citée, et l'analyse par Giuliana Costa-Colajanni des sept parties qui composent le texte, «point d'arrivée formel d'une période d'expérimentation de nouveaux modèles narratifs ${ }^{101}$. Sur L'Acacia nous disposons du texte de Guido Neri, déjà mentionné, et du compte rendu de Gianni Poli, qui voit dans le roman à la fois "un témoignage et un testament ", tout en relevant son "formalisme baroque qui nie l'immédiateté de la communication ${ }^{102}$. Un seul essai a été publié sur La Corde raide: celui de Marco Nuti, qui analyse la présence de Cézanne dans ce texte et dans l'œuvre de Claude Simon, et qui montre que sa réflexion esthétique est indissociable d'une ontologie ${ }^{103}$. Létude du Vent par Graziano Benelli tourne autour du concept d'étrangeté tant au niveau thématique qu'au niveau de l'écriture $^{104}$. Sur Le Palace, il faut mentionner le travail de Raul Lunardi, qui

98. Jean Rousset, "Histoire de Claude Simon : les cartes postales ", Studi di letteratura francese, VIII, «Il romanzo in discussione », 1982, p. 28-33 et Giuliana Costa-Colajanni, "Il cronotopo delle cartoline postali in Histoire di Claude Simon ", dans Il luogo e la distanza, sous la direction de G. Costa Colajanni et Giovanni Saverio Santangelo, Palerme, Palumbo, 1995, p. 65-82.

99. Andrea Calì, " Dove il narratore ?", in Id., Saggi sul racconto autoreferenziale, Lecce, Adratica Editrice Salentina, 1983, p. 95-113.

100. Alberto Casadei, "Ancora su La Bataille de Pharsale e le arti figurative", Francofonia, XVII, 32, printemps 1997, p. 77-81.

101. Giuliana Costa-Colajanni, "Leçon de choses di Claude Simon. Storie e parole in fuga e contrappunto ", dans Scritture e forme del Novecento francese, sous la direction de G. Costa-Colajanni et Maria-Teresa Russo, Palerme, Flaccovio, 2000, p. $29-48$; p. 47.

102. Gianni Poli, «Avanzando sulle sabbie mobili. L'acacia di Claude Simon ", Il Ponte, 51, 5, 1995, p. 119-122; p. 119.

103. Marco Nuti, «Entre lisible et visible : convergences cézaniennes chez Claude Simon ", dans Id., Et in pictura fabulator. Paul Cézanne et le dialogue créateur entre peinture, littérature et philosophie, de Balzac à Maldiney, Fasano, Schena / Paris, Baudry, 2008, p. 123-135.

104. Graziano Benelli, "L'étrangère: riflessioni su Le vent di Claude Simon », dans 
commente différents aspects de l'art de Claude Simon en comparant sa description des funérailles et celle qui se trouve dans LInsurgé de Vallès ${ }^{105}$. Mireille Calle-Gruber interprète Les Géorgiques à travers les lois de l'hétérogène, du cyclique et du quiproquo ${ }^{106}$, tandis que l'étude d'Andrea Calì sur Les Corps conducteurs porte sur les concepts de littérature et de paralittérature ${ }^{107}$. Sur La Chevelure de Bérénice nous disposons de la postface de Guido Neri, déjà citée, et, sur le Discours de Stockholm, du compte rendu de Paolo Budini, qui parle de son ton " ironiquement simple " d'où " émergent des dénonciations fortes " ${ }^{108}$. Sur L'Invitation il faut citer, outre le commentaire de Marco Ferri qui accompagne la traduction italienne, cité plus haut, l'essai de Paolo Budini, qui considère la genèse du texte, son référent réel et son référent exotique imaginaire, soulignant les procédés de distanciation qui le caractérisent ${ }^{109}$. Enfin, dans son étude de Photographies 1937-1970, Mireille Calle-Gruber montre " en quoi la manière simonienne de travailler la photographie offre une compréhension rétrospective de son art du roman au travail de la mémoire et du dépôt des traces ${ }^{110}$.

Si le critère quantitatif désigne La Route des Flandres comme l'œuvre la plus étudiée (c'est aussi l'œuvre qui a connu le plus de rééditions en Italie), le critère chronologique montre qu' Histoire a fait l'objet d'une attention précoce et constante, alors que Leçon

Lo straniero. Atti del Convegno di Studi (Cagliari, 16-19 novembre 1994), sous la direction de Mario Domenichelli et Pino Fasano, Rome, Bulzoni, 1997, t. II, p. 873-880.

105. Raul Lunardi, "I piccioni di Simon (Il 'Palace') ", Studi Urbinati. Linguistica, Letteratura, Arte, LIX, 1986, p. 107-122.

106. Mireille Calle-Gruber, "Sur les brisés du roman », Micromégas, VIII, 1, janvier-avril 1981, p. 108-113.

107. Andra Calì, "Littérature et paralittérature dans Les Corps conducteurs de Claude Simon ", dans Filosofare dialogando. Studi e testimonianze per Angelo Prontera, sous la direction d'Andra Calì, Jean-François Durand, Marisa Forcina et Pia Vergine, Lecce, Edizioni Milella, 2002, p. 155-161.

108. Paolo Budini, compte rendu de Claude Simon, Discours de Stockholm, Francofonia, IX, 16, printemps 1989, p. 123-124; p. 123.

109. Id., "Le réel et l'écriture dans L'invitation de Claude Simon », Francofonia, IX, 17, automne 1989, p. 3-20.

110. Mireille Calle-Gruber, "Un pas de plus. Photobiographies de Claude Simon ", dans Letteratura e fotografia, sous la direction d'Anna Dolfi, Rome, Bulzoni, t. II, 2005, p. 129-146; p. 129-130. 
de choses, traduite tardivement, a été étudiée avec beaucoup de retard.

En complétant ces données par l'examen de la bibliographie complète, on peut formuler deux autres remarques. En premier lieu, force est de constater que, à l'exception de la Correspondance avec Jean Dubuffet et d'Archipel et Nord, la critique italienne a abordé la totalité de l'œuvre simonienne, mais avec des textes moins fréquentés, comme Le Jardin des plantes (cité dans quelques essais) et surtout Le Tramway, dont on trouve des comptes rendus dans la presse, mais non des études critiques approfondies. En second lieu, il faut souligner la prédilection de la critique italienne pour La Route des Flandres et pour Histoire. Le premier texte, considéré comme l'un des romans les plus significatifs et les plus représentatifs ${ }^{111}$, passe pour inaugurer, en 1960, "la décennie des œuvres majeures de Claude Simon " ${ }^{12}$, alors que le second est considéré comme le « livre-pivot de l'œuvre entière, le plus dense et le plus travaillé " ${ }^{113}$.

Mais il est vrai aussi que, ces dernières années, l'attention semble se déplacer vers les œuvres des décennies suivantes, en particulier celles qui témoignent de l'intérêt du romancier pour d'autres langages, notamment la photographie et le cinéma, comme le confirment deux articles et deux monographies proposant une lecture intersémiotique, parus entre 2003 et 2005.

\section{Claude Simon dans la sitographie italienne}

Je voudrais conclure cette étude de la réception de l'œuvre de Claude Simon par une brève description de la sitographie en langue italienne. Sur l'écrivain, le réseau Internet fournit des informations hétérogènes et variées : des pages de Wikipedia à l'encyclopédie Treccani, des catalogues de bibliothèques aux sites de librairies, des archives des grands quotidiens à celles de la télévision, des pages didactiques sur la littérature française aux textes critiques publiés

111. Respectivement par Pierluigi Ligas, «L'avventura del racconto », cit., p. 120 et Manuela Raccanello et Graziano Benelli, op. cit., p. 28.

112. Anna Paola Mossetto, "Claude Simon ", dans Claude Simon, Le opere, cit., p. XXVII.

113. Ibid., p. XXX. 
en ligne. Le réseau Internet permet aussi de dresser la bibliographie secondaire - y compris les mémoires de maîtrise soutenus dans les universités italiennes ${ }^{114}$ et une thèse de doctorat récente ${ }^{115}$ - et de prendre connaissance de certaines études critiques.

Parmi ces études, il faut rappeler celle de Giuseppe Zuccarino ${ }^{116}$, qui analyse la présence constante de "choses écrites " (calepins, cartes postales, lettres et manuscrits) dans les romans simoniens (en particulier dans L'Herbe, Histoire, Les Géorgiques et L'Acacia). Ces " choses écrites " font l'objet de descriptions méticuleuses qui, d'une part, révèlent la participation affective du narrateur et, d'autre part, attirent l'attention du lecteur sur la matérialité de l'écriture. Deux aspects qui se retrouvent dans les pages qui, à la fin de L'Acacia et de La Bataille de Pharsale, décrivent l'écrivain en train d'écrire (geste qui apparaît aussi dans les dessins réalisés par l'auteur). Une nouvelle fois, c'est l'acte d'écrire qui est mis en évidence pour illustrer, comme le rappelle la préface d'Orion aveugle, l'aventure d'un narrateur qui découvre le monde dans et à travers l'écriture.

Outre l'essai de Giuseppe Zuccarino, on trouvera sur Internet deux textes critiques qui comparent les romans de Claude Simon et ceux de Jean Rouaud d'un point de vue thématique et formel. L'étude de Carine Trevisan, consacrée à la Grande Guerre, rappelle "l'obsession simonienne pour le corps absent du père " et consi-

114. On rappellera entre autres, suivant l'ordre chronologique, les mémoires de Lorenza Morelli, Claude Simon. Contraddizioni e continuità, sous la direction d'Enea Balmas, Université de Padoue, 1974 ; Fabio Prescimone, Le Vent di Claude Simon. Ricostruzione del protagonista, sous la direction de Simonetta Micale, Université de Messine, 2000-2001 ; Annamaria Martinolli, Le Jardin des plantes de Claude Simon : proposta di traduzione di alcuni passi dell'opera, sous la direction de Manuela Raccanello, Université de Trieste, 2004-2005 ; Rosario Antoci, Frammentazione del testo e dell'individualità in Histoire di Claude Simon, sous la direction d'Hélène de Jacquelot et d'Arrigo Stara, Université de Pise, 2005.

115. Luigi Vallebona, Narrare il contatto col mondo : percezione e memoria nell'opera narrativa di Claude Simon e Juan José Saer, Université de Macerata, Dipartimento di Lingue e Letterature moderne, Corso di Dottorato di Ricerca in Lingue e letterature comparate, ciclo XXIII, 2010.

116. Giuseppe Zuccarino, "Claude Simon e la mano che scrive ", La dimora del tempo sospeso, 28 août 2010 ; http://rebstein.wordpress.com/2010/08/28/claudesimon-e-la-mano-che-scrive/. 
dère les deux romanciers comme des " orphelins ou descendants d'orphelins [qui] abordent dans leurs récits l'amputation d'une partie des racines de leur identité " pour " rendre à ceux qui ont été effacés par l'histoire un corps et un visage singuliers $»^{117}$. Le texte de Massimiliano Badiali souligne également la " recherche du passé, d'une histoire familiale plus qu'individuelle ", commune aux deux auteurs, et décrit leur écriture comme un magma " où le lecteur doit trouver le fil d'Ariane dans le labyrinthe évocateur du souvenir $»^{118}$.

Consacrée au Jardin des plantes, un article de Massimo Leone explore le concept du quinconce en tant que possible médiation entre deux conceptions antithétiques du paysage, l'une pragmatique et l'autre esthétique. En particulier, l'auteur montre comment, après avoir été la figure par laquelle l'homme essaya de dominer la nature du paysage et du langage (dans Quintilien), le quinconce est devenu une sorte de piège dans l'architecture de mort du dernier roman de G.W. Sebald, tandis que le narrateur du Jardin simonien, tout en essayant de "domestiquer la mémoire à travers l'écriture, comme un jardinier ordonne l'exubérance de la nature ", laisse " pousser la mauvaise herbe parmi les fleurs pour témoigner que tout n'est pas forme et cohérence dans la vie, et que la mémoire de l'homme sincère ressemble plus à un jardin de campagne, à un paysage accidenté de fossés et de bourbiers, qu’au parc royal de Versailles ». Ainsi la forme typographique du texte apparait-elle comme « l'empreinte, la gravure, de cet excès de la nature/mémoire, qui sème les fragments de la pensée comme les grains dans les sillons d'un champ irrégulièrement labouré $\aleph^{119}$.

Enfin, parmi les sites qui font mention de Claude Simon, il faut

117. "La Grande Guerra : la memoria in lutto ", trad. par M. G. G., Il Manifesto / Le Monde diplomatique, novembre 2001 ; http://www.monde-diplomatique.it/LeMonde-archivio/Novembre-2001/pagina.php? $\operatorname{cosa}=0111 \mathrm{~lm} 22.01$. html\&Titolo=La\%20Grande\%20Guerra:\%20la\%20memoria\%20in\%20lutto.

118. «Jean Rouaud : riflessioni sul romanzo »; http://www.massimilianobadiali.it/ confrontiRouaud.htm.

119. "Quinconce e altre griglie. Il paesaggio fra estetica ed economia ", Golem l'Indispensabile, 1 septembre 2001 ; http://www.golemindispensabile.it/index. php?_idnodo=70438_idfrm=107. 
signaler les multiples références à deux figures de la culture italienne : Mario Dondero et Gastone Novelli.

Auteur d'une riche documentation photographique sur le monde intellectuel et artistique et sur la réalité sociale et politique de la deuxième moitié du XXe siècle, Mario Dondero fut l'un des fondateurs du photojournalisme italien. Collaborateur de plusieurs organes de presse français (Le Monde, Le Nouvel Observateur, Le Figaro), il fut l'un des protagonistes de "La vie aigre » chantée par Luciano Bianciardi et se lia d'amitié avec des écrivains, des cinéastes et des acteurs italiens et français. Cet « intellectuel de l'image " ${ }^{120}$, considéré comme le " douanier Rousseau de la photographie ", comme le " chanteur d'une épopée du quotidien $»^{121}$, a définitivement associé son nom à celui de Claude Simon et à l'histoire du Nouveau Roman en photographiant, le 16 octobre 1959, devant le siège des Éditions de Minuit, les écrivains du Nouveau Roman en compagnie de leur éditeur, Jérôme Lindon. L'image, restée célèbre, réunissait Alain Robbe-Grillet, Claude Simon, Claude Mauriac, Robert Pinget, Samuel Beckett, Nathalie Sarraute et Claude Ollier. Interrogé par la télévision italienne, Alain Robbe-Grillet déclara que l'auteur de cette « image très bizarre " et " très réussie " était l'inventeur du Nouveau Roman : "Chose curieuse : le groupe a été inventé par un photographe italien [...]. Nous devons donc notre célébrité à Mario Dondero " ${ }^{122}$.

Dans la même émission, le photographe italien s'expliqua sur les circonstances dans lesquelles fut prise cette image devenue symbolique : «Ce qui s'est passé, c'est que j’ai demandé à Jérôme Lindon, qui était l'éditeur de Minuit [...], s'il pouvait réunir ces écrivains ; accueillant cette idée avec enthousiasme, il fit aussi venir Samuel Beckett, qui était absolument allergique à la photographie. La force

120. Marco Pinna, "Mario Dondero, l'intellettuale dell'immagine ", $\mathrm{Na}$ tional Geographic Italia, 2 juin 2010 ; http://www.nationalgeographic.it/ fotografia/2010/06/02/news/dondero_1_intellettuale_della_fotografia_ intervista_a-28918/index.html.

121. Selon son ami et collègue Danilo De Marco, "Mario Dondero : l'angelo necessario " (2008) ; http://www.danilodemarco.it/parole/danilo-de-marco/langelo-necessario/.

122. Archives Rai - Scrittori per un anno http://www.scrittoriperunanno.rai.it/video.asp?currentId=222. 
de l'image tient peut-être aussi à la façon de photographier ces personnages, comme s'ils étaient là presque par hasard, au lieu de poser à la façon d'une équipe de football. ".

Passée à l'histoire, l'image a suscité d'innombrables commentaires et lectures critiques. Raffaele Scolari y voit " une photo d'une simplicité désarmante : c'est comme si le photographe-flâneur, qui passait là par hasard, avait saisi sans intentions particulières un groupe de personnes pendant la pause d'une réunion ». Et d'ajouter : " la célèbre photo de Dondero a fait du Nouveau Roman un événement qui se renouvelle chaque fois qu'on la regarde ; [...] elle est un éclair qui rend visible, presque palpable, l'existence d'une sorte de communauté ou de convergence entre des personnalités très différentes $»^{123}$.

Pour son auteur, cette " photo de classe " fut surtout un "facteur de relations humaines ", car c'est à cette occasion que Mario Dondero se lia d'amitié avec Claude Simon et Alain Robbe-Grillet, comme lui-même le rappelle dans une interview de $2009^{124}$.

L'artiste d'origine juive Gastone Novelli, qui survécu à la déportation et disparut en 1968 à l'âge de 43 ans, fut immortalisé, lui aussi, par l'objectif de Mario Dondero. Lié aux principaux représentants des avant-gardes littéraires et artistiques, en Italie et à l'étranger, il fréquenta Tristan Tzara, André Masson, Man Ray et Hans Arp, ainsi que les écrivains Samuel Beckett, Georges Bataille, Pierre Klossowski et, bien sûr, Claude Simon, qui le fit apparaître dans le Jardin des plantes ${ }^{125}$ et le cita aussi dans le Discours prononcé à

123. Raffaele Scolari, « La fotografia che fa l'evento. Mario Dondero al Rivellino " ; http://docs.google.com/viewer?url=http://territoridelpresente.ch/images/ documenti/fotografia.pdf. Très différente l'interprétation d'Anne Simonin, selon qui « l'historique de la photo est moins l'histoire d'une représentation que celle de la 'présentation de soi' des Éditions de Minuit ayant réussi à s'imposer comme une maison d'édition subversive "; "La photo du Nouveau Roman. Tentative d'interprétation d'un instantané ", Politix, 3, 10-11, deuxième et troisième trimestre 1990, p. $45-52$ (http://www.persee.fr/web/revues/home/prescript/article/ polix_0295-2319_1990_num_3_10_2121).

124. Cf. Gianluigi Colin, "Così inventai il Nouveau Roman ", Il Corriere della Sera, 27 décembre 2009, p. 35 ; http://archiviostorico.corriere.it/2009/dicembre/27/Cosi_inventai_Nouveau_Roman_co_9_091227063.shtml.

125. Ce rapport Simon-Novelli a été analysé, entre autres, par Brigitte Ferrato- 
Bologne en 1989. La sitographie italienne rend compte de leur collaboration, en rappelant le dialogue nouveau qui s'établit, à cette occasion, entre peinture et écriture ${ }^{126}$. Et si le lecteur italien n’a pas accès aux pages du Jardin des plantes, il peut néanmoins lire le texte simonien consacré à "Novelli e il problema del linguaggio ", qui présente la vie et l'œuvre de l'artiste, en soulignant la synthèse réalisée par ses deux Salles de Musée, où il essaie, tout en étant conscient de la vanité de cette tentative, de "capter le monde à travers cette collection de signes, cet alphabet de 'choses aimées' $»^{127}$.

Nous conclurons par ces mots de Claude Simon évoquant l'œuvre de son ami peintre, car l'hommage rendu par l'écrivain au peintre italien nous parle aussi de l'aventure simonienne dans et par le langage :

Sans cesse, irrémédiablement et inexorablement, la vie échappe à notre désir de connaissance, de possession. Nous ne pourrons en saisir que d'éphémères lambeaux, des fragments. Des formes, des mots qui se désagrègent, s'émiettent, puis se recomposent selon le caprice de leurs propres lois, [...] : là est toute la magie du Verbe, du langage.

\section{$[\ldots]$}

Le langage n'est que fulguration, brève étincelle, bribes captées. Le monde splendide, à peine saisi, se dérobe, s'échappe, se reforme, sans cesse recommencé, sans cesse prêt à recommencer. C'est la perpétuelle

Combe, "Simon et Novelli : l'image de la lettre ", dans "Le Jardin des plantes" de Claude Simon, Actes du Colloque de Perpignan (27 mars 1999), études réunies par Jean-Yves Laurichesse, Cahiers de l'Université de Perpignan, 30, 2000, p. 103-118 et "De Cézanne à Novelli, la figure du peintre dans l'œuvre de Claude Simon ", dans Les Images chez Claude Simon : des mots pour le voir, textes réunis par Stéphane Bikialo et Catherine Rannoux, La Licorne, 71, 2004, p. 169-180 et par Jean-Yves Laurichesse, "Orion aveugle dans la forêt amazonienne. L'aventure de Novelli dans le Jardin des plantes de Claude Simon ", dans Claude Simon et Le Jardin des plantes, études réunies par Sjef Houppermans, Amsterdam, Rodopi, 2001, p. 45-61.

126. On rappellera, entre autres, le site de la Fondazione Arnaldo Pomodoro : http://www.Fondazionearnaldopomodoro.it/artisti.php?act=biografia\&id_artis$\mathrm{ta}=16$ et http://www.accart.it/ga_no.htm, site de la Galleria Antonella Cattani contemporary art de Bolzano.

127. Claude Simon, "Novelli e il problema del linguaggio ", dans Novelli, sous la direction de Zeno Birolli, Milan, Feltrinelli, 1976, p. 85-87 ; p. 87. 
étincelle, la perpétuelle surprise, la perpétuelle remise en question, le perpétuel mirage, le perpétuel combat, dont la conscience de ce qu'il a de vain constitue pour l'homme qui toutefois l'engage le plus beau titre de grandeur. ${ }^{128}$ 